\title{
GEOREFERENCING OF TLS DATA FOR INDUSTRIAL INDOOR COMPLEX SCENES: BEYOND CURRENT SOLUTIONS
}

\author{
Jean-François Hullo*a b ${ }^{\text {, Pierre Grussenmeyer }}{ }^{a}$, Tania Landes ${ }^{a}$ and Guillaume Thibault ${ }^{\text {c }}$ \\ ${ }^{a}$ The Image Sciences, Computer Sciences and Remote Sensing Laboratory INSA, 67000 Strasbourg, France \\ ${ }^{\mathrm{b}}$ EDF R\&D, 92141 Clamart, France \\ ${ }^{c}$ Laboratoire de Physiologie de la Perception et de l'Action, Collège de France, UMR 7152, CNRS, Paris, France \\ (jean-francois.hullo, pierre.grussenmeyer, tania.landes)@insa-strasbourg.fr, guillaume.thibault@edf.fr
}

\section{Commission V/3}

KEY WORDS: TLS, primitive-based registration, indoor georeferencing, industrial installations, geolocation

\begin{abstract}
:
Current Terrestrial Laser Scanners (TLS) allow fast acquisitions of many dense point clouds. This technology is widely used within industrial complex scenes. The precise georeferencing of all the per-station point clouds is a crucial stage. Nowadays, the use of targets or tacheometry for precise georeferencing is time-consuming and then limits the number of surveys. The purpose of this article is to present and analyse a primitive based registration using points, lines and planes automatically extracted and paired in the station point clouds. Our tests highlight the advantages and the current limitations of this approach. We also discuss the recent, and probably future, improvements of indoor geolocation systems as possible ways of research for the georeferencing of TLS data into industrial complex scenes.
\end{abstract}

\section{INTRODUCTION}

Several industrial applications require as-built models of the whole or part of their installations (mostly indoor and complex as illustrated in figure 1). The number of surveys increases, because the tools for analysis and simulations based on 3D data are becoming more numerous and powerful. The utility of the Terrestrial Laser Scanner (TLS) for 3D acquisition of industrial scenes, as point clouds, is now admitted. The current resolution and speed of the TLS make it possible to consider wide campaigns of dense and accurate measurement, i.e. hundreds of scans, or even more.

Though the GPS + IMU combination solved many problems of georeferencing for outdoor measurements, indoor georeferencing of TLS data remains a research topic.

Our contributions in this paper are the followings:

- A review of several registration techniques for indoor complex installations, cf. section 2;

- A description of a primitive-based registration algorithm, cf. section 3 .

- A test on two typical scenes: description, results and discussion, cf. section 4

\section{3D POINT CLOUD ACQUISITION OF INDUSTRIAL SCENES}

\subsection{Current effective best practices}

Constraints. Due to functional constraints of the installations, the time allowed for the acquisition of TLS data can be very short. However, the position accuracy of the global point cloud after registration must typically reach $1-2 \mathrm{~cm}$. Georeferencing is frequently required to make data fusion and retrieval easier.

Best practices. Currently, inside wide and complex industrial scenes, the common practice to georeference station point clouds is based on tacheometry. Since only few known points exist, surveyors have to traverse across rooms and corridors to establish

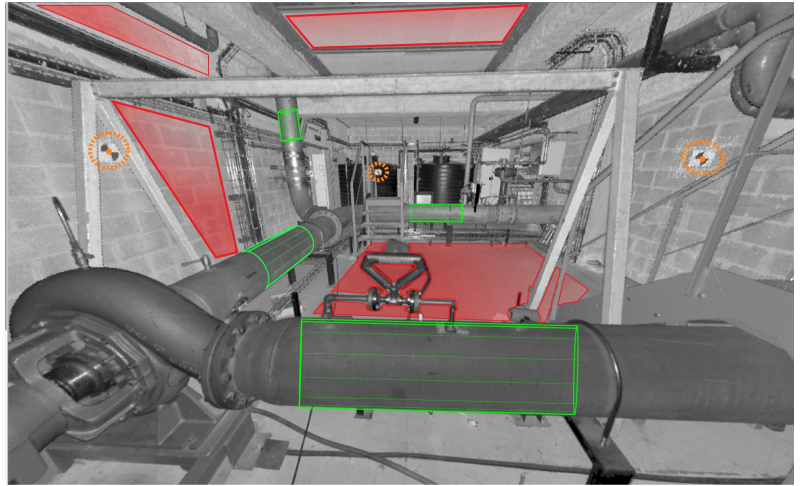

Figure 1: Typical station-based view of an industrial complex scene. Grey level: point cloud coded in intensity. Colour: reconstructed 3D primitives.

a control network. This method is time consuming with regard to the time of scanning but ensures the quality of the results. With the created network, surveyors are free to use targets to georeference directly, as discussed in (Lichti and Gordon, 2004) and (Scaioni, 2005), or indirectly, as presented in (Balzani et al., 2001).

Improvements. We aim at improving the previous methods in order to reduce the time of on-site acquisition and, as often as possible, to georeference without targets and total station.

\subsection{Related works and selected approach}

Registration of point clouds haveshe design of the hardware and by the capacities of the processing software. As (Reshetyuk, 2009) explains in the introduction of his $\mathrm{PhD}$ work: "The reason for using the indirect georeferencing approach was the design of the scanner hardware. Most of the earlier scanner models lacked the appropriate facilities for direct georeferencing". We will first consider the advances in the field of relative registration and thereafter the advances in georeferencing. 
ICP. Many communities (such as Robotics, Computer Vision or reverse Mechanical Engineering) have worked on the registration of point clouds. With the ICP, (Besl and Mckay, 1992) have defined a cloud-to-cloud approach which has since been widely used and adapted. Despite its improvements, this method cannot be used in our applications because: (a) the industrial scenes can be very regular; (b) the differences between point clouds from several stations can be large; and (c) the great number of stations and points per stations leads us to work with huge amount of data. Under those conditions, the accuracy of $1-2 \mathrm{~cm}$ on the complete dataset cannot be reach by using the ICP or its current variants.

Keypoints. Targets are commonly used as keypoints for either registration or georeferencing of point clouds. In this case, the geometry of the network has to be designed before the acquisition of TLS data. Another approach is presented in (Barnea and Filin, 2008): tiepoints are automatically extracted from range images using local descriptors and a RANSAC is performed. In our configuration, few tiepoints can be extracted because the scene is composed of planar or smooth objects, mainly grey or metallic. Without enough details in geometry or texture of the scene, this approach does not lead to the required accuracy.

Geometric features for Photogrammetry. The feature-based approach is another method to overcome the targets for the registration. Since 1980, several researchers in digital photogrammetry have argued for the use of 2D linear features instead of points, as (Schenk, 2004) explains. For terrestrial photogrammetric applications, linear features are commonly used for matching or pose (position and orientation of the sensor) estimation. Projective geometry fully exploits the linear features, as presented and detailed in (Hartley and Zisserman, 2000) and (Forsyth and Ponce, 2002). This method for the registration of 2D images inspired the feature-based registration of 3D TLS data.

Geometric features for TLS. The detection of linear features in TLS data, and their use for registration, is presented in (Jaw and Chuang, 2008b). (Rabbani and van den Heuvel, 2005) introduced a flexible registration using simultaneously several 3D geometric entities (points, lines and planes) from 3D reconstructed primitives (mainly cylinder, sphere and plane). This approach was improved by (Jaw and Chuang, 2008a) by defining several constraints for the functional model and also a more complete stochastic model. An adaptation of those two works will be presented in the section 3 .

Prerequisites. Since TLS data are not directly used for the registration in the feature-based approach, we first have to consider the stage of extraction and then the stage of pairing. Latest improvements in the automatic extraction of 3D primitives in point clouds, (Schnabel et al., 2007) and (Rabbani et al., 2007), allow us to rely on the high level of automation and reliability of those methods. Pairing the 3D entities is a key point of the method. Indeed, the principle of bundle adjustment does not allow any wrong pairing. Therefore, it is important to check the exactness of pairing upstream of estimation of the poses. To correctly pair the entities, (Rabbani and van den Heuvel, 2005) constraint the search by using the geometric attributes of 3D primitives such as radius for cylinders or spheres. (Thapa, 2009) adds a semantic architectural constraint to pair windows and walls. The search interval for pairing is also limited by the uncertainty of the approximate pose parameters of the stations. Although it does not ensure the existence of a solution for pairing problem, the constraints on the search interval can drastically reduce the number of wrong pairs.

\subsection{Using as many sources of information as possible}

We have a corpus of information including data and uncertainty on it. We want to maximally exploit this corpus in the registration. The method needs to be able to integrate many types of inputs:

- Approximate values of scan positions and orientations measured with geolocation devices such as INS or odometer;

- Bi-axial compensator;

- 2D maps of the civil works;

- A priori 3D CAD model of the installation, especially civils;

- Partial, less dense and/or non-updated previous point cloud;

- Images or panoramas from calibrated or uncalibrated cameras;

- Knowledge and surveyor's estimation of his own position and heading.

By integrating all this information, the purpose is to help to calculate the pose parameters, to limit the search space of the unknown parameters and to improve the accuracy of georeferencing.

\section{A PRIMITIVE-BASED REGISTRATION ALGORITHM}

In this section, we present a feature-based registration approach, inspired by (Rabbani et al., 2007) and (Jaw and Chuang, 2008a).

\subsection{Principle}

a. before adjustment, with approximated values.

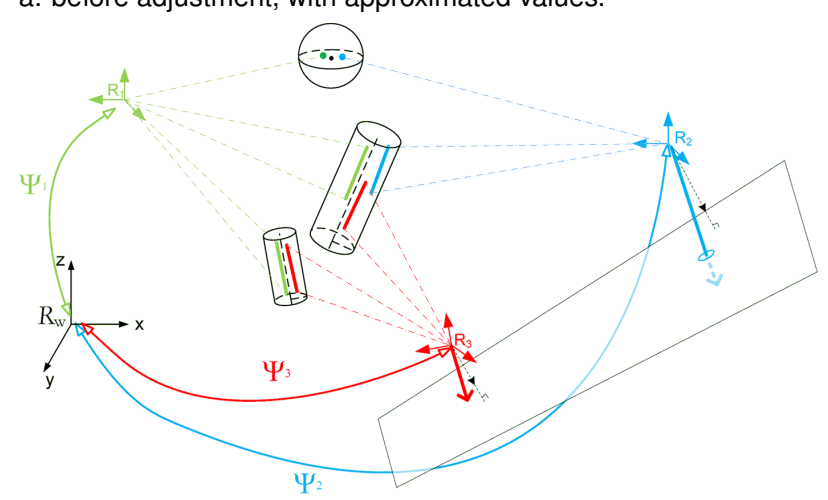

b. after adjustment, constraints residuals minimized.

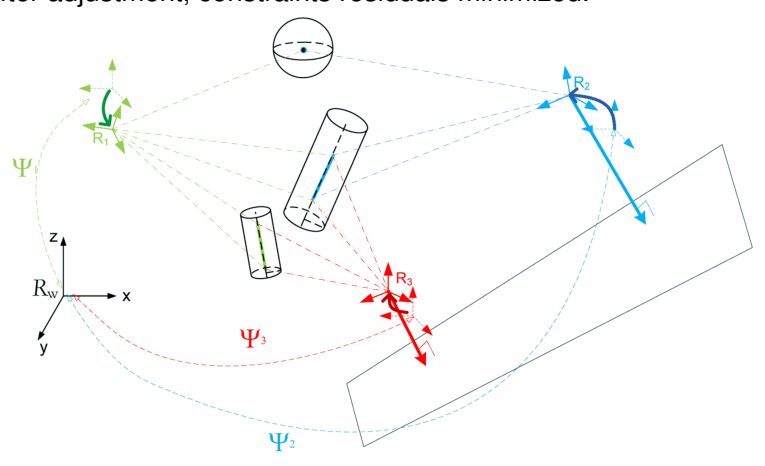

Figure 2: Principle of the current feature-based registration. In the scene: 3 stations, 2 lines, 1 point and 1 plane.

The idea is to use geometric entities (points, lines, planes) of reconstructed 3D primitives from several scan stations, to simultaneously register them, as illustrated in figure 2 . 
First, we have to choose an efficient parameterisation of the pose (position and orientation) of the stations. Efficient means here that we want to avoid dependency on the unknown parameters and to fit the geometric model to the nature of the uncertainty of the approximate values (measured with a geolocation system). Similarly, the choice of the parameterisation of the 3D primitives has to fit as well as possible the uncertainty of the point clouds and primitives reconstruction.

Thus, the stochastic and the functional (geometric) model are developed using the technical specifications of the equipment. The definition of the constraints depending on the nature of the errors is also studied.

\subsection{Parameterisation of the transformations}

Definitions. We first define the global three dimensional Cartesian coordinate system ${ }^{g} \mathcal{R}=\{O,[\vec{x}, \vec{y}, \vec{z}]\}$, right-handed. The pose parameters $\boldsymbol{\Psi}_{s}$ of a station $\boldsymbol{s}$ are defined as the parameters of the frame transformation of any point from ${ }^{g} \mathcal{R}$ to the attached frame of the station ${ }^{s} \mathcal{R}$, then $\boldsymbol{\Psi}_{s}={ }^{g \rightarrow s} \boldsymbol{\Psi}$. The translation $\boldsymbol{t}_{s}={ }^{g \rightarrow s} \boldsymbol{t}$ is simply a three coordinate vector $\left[t_{x} t_{y} t_{z}\right]$. As detailed in (Förstner and Wrobel, 2004) and studied in (Nüchter et al., 2010), many parameterisations can be used for rotations. In order to fit the hardware design of TLS, we use the three concatenated rotations, of angles $\{\omega \phi \kappa\}$, for a frame transformation respectively around $x, y$ and $z$. This means that the $3 \times 3$ rotation matrix ${ }^{g \rightarrow s} \boldsymbol{M}$ used for frame transformation of a point from ${ }^{g} \mathcal{R}$ to ${ }^{s} \mathcal{R}$ can be evaluated using the matrix relation ${ }^{g \rightarrow s} \boldsymbol{M}=$ $\boldsymbol{M}_{-\omega} \boldsymbol{M}_{-\phi} \boldsymbol{M}_{-\kappa}$.

Approximate values of translations. In our configuration, we always have a 2D map of civil works and sometimes of major equipments and pipes. Due to construction tolerances, the errors of position of the walls can reach $5 \mathrm{~cm}$ in the worst case. Due local modifications, maps of equipments can be locally incomplete or not up-to-date. But this information can help the operator to approximate the position of the stations. For the $z$ value, the height of the instrument is added to the altitude of the groundfloor. For the $(x, y)$ value, the surveyor indicates the position of the station on a drawing of the civils.

To estimate the ability of surveyors to locate a ground point on a local map of civils, we carried out a psycho-experimental study whose results of this experiment allow us to make quantitative assumptions on the uncertainty of this type of approximations. Fifteen students in topography of the INSA Strasbourg were tested. The experiment took place in the basement of the INSA Strasbourg, considered as an indoor facility. Each student was guided through the building, from ground point to ground point. For each one of the 15 ground points, the student had to indicate on a local map the position of this ground point. The distance errors of the pointed locations is studied.

One student was rejected because of particularly incoherent results. For the other 14 students, 75 percents of the points were closer than $50 \mathrm{~cm}$ to the true position. Only 4 percents of the answers were further than $1.5 \mathrm{~m}$ (fault).

In conclusion: stand.dev. $(x, y)=50 \mathrm{~cm}$ and stand.dev. $(z)=$ $15 \mathrm{~cm}$.

Approximate values of rotations. Currently, most laser scanners used for the acquisition of industrial scenes have a bi-axial compensator with range from $0.08^{\circ}$ to $10^{\circ}$. Their accuracy varies from $0.015^{\circ}$ to $0.00008^{\circ}$, depending on brands and models. Consequently, we can set, through calibration, $(\omega, \phi)=0$ with an uncertainty given by the manufacturer or by calibration. This assumption can also be found in (Reshetyuk, 2009). Regarding $\kappa$, since $(\omega, \phi) \bumpeq 0$, we can easily get an approximate value of the $\kappa$ through the heading value of INS or IMU. However, our own experiments on a Crossbow AHRS 440 have shown that this heading value can be strongly disturbed (up to $20^{\circ}$ ) with the proximity ( 0 to 1 meter) of huge steel materials (such as pipes, turbines and large metallic objects). These metallic objects are commonly present in industrial scenes. Without a more reliable system for heading approximation, the registration algorithm must handle poor approximations for $\kappa$.

Selected parameterisation for the transformations. The previous assumptions on the approximated values of the pose parameters lead us to choose the following parameterisation of the transformations, involving 4 parameters: 3 translations and only 1 rotation. Consequently: $\Psi_{s}=\left\{\kappa t_{x} t_{y} t_{z}\right\}$.

\subsection{Representation of the objects}

In the scene, we can extract several 3D primitives: spheres (targets), cylinders (pipes), polyhedron (walls and boxes) and possibly cones or torus. For the registration, we only consider 3 types of geometric entities: points, lines and planes. Typically we use points from targets or sphere centres, lines from cylinder axis and plane from polyhedron faces or walls.

Points. A point $\mathrm{Pt}$ is represented by its 3 Cartesian coordinates. For convenience, we use normalized homogeneous coordinates.

Lines. Many representations can be used. A line has only 4 degrees of freedom, but most representations involve 6 parameters to avoid singularities. In our implementation we use the convenient normalized Plücker coordinates as defined in (Förstner and Wrobel, 2004) for the definition of the constraints. We mention (Schenk, 2004) who uses a 4 parameters representation, 2 orientation and 2 positional parameters, which allows a better stochastic interpretation of line parameters but does not avoid special behaviour for horizontal and vertical lines, mainly represented in our configuration.

Finally, we represent a line $\mathrm{Li}$ with two 3D vectors: $\boldsymbol{u}$, its unit directional vector and $v$, a normal vector of the plane through the line and origin with length equal to the distance of the 3D line to the origin. Using two arbitrary distinct points $(x, y)$ with Cartesian coordinates $(\boldsymbol{x}, \boldsymbol{y})$, we can easily compute $\boldsymbol{u}$ and $\boldsymbol{v}$. The closest point to origin, $\boldsymbol{w}$, can also be easily computed. $\times$ is the vector product.

$$
\mathrm{Li}=\mathrm{x} \wedge \mathrm{y}:=\left[\begin{array}{l}
\boldsymbol{y}-\boldsymbol{x} \\
\boldsymbol{x} \times \boldsymbol{y}
\end{array}\right]=|\boldsymbol{y}-\boldsymbol{x}|\left[\begin{array}{l}
\boldsymbol{u} \\
\boldsymbol{v}
\end{array}\right] \quad \boldsymbol{w}=\boldsymbol{u} \times \boldsymbol{v}
$$

Planes. For a plane PI, we use the Hessian normal form using unit normal vector $\boldsymbol{n}=\left[n_{1} n_{2} n_{3}\right]$ and the distance $\boldsymbol{d}$ from the origin. In summary:

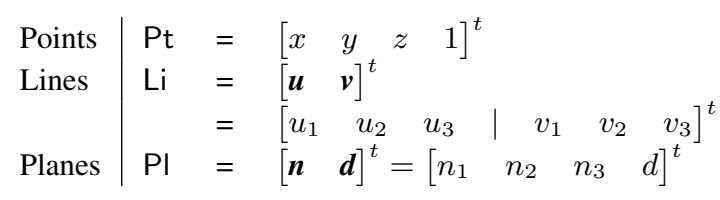

\subsection{Definition of the constraints}

For the following relations, we use as notation for the entities: ${ }^{\text {Frame }}{ }^{T_{\text {Type }}}$ Station of measure $_{\text {ID en }}$. For example line number 15 measured in the station 101 and expressed in the global frame has to be identical to line number 15 measured in the station 106 expressed in the global frame: ${ }^{g} \mathrm{Li}_{101}^{15}={ }^{g} \mathrm{Li}_{106}^{15}$

When two 3D primitives are paired, we have to bring them into a common frame to apply constraints on their properties. For the 
study of frame transformations, let's consider two frames ${ }^{1} \mathcal{R}$ and ${ }^{2} \mathcal{R}$ and the parameters ${ }^{1 \rightarrow 2} \boldsymbol{\Psi}={ }^{1 \rightarrow 2}\left\{\kappa t_{x} t_{y} t_{z}\right\}$ of the frame transformation from ${ }^{1} \mathcal{R}$ to ${ }^{2} \mathcal{R}$. The rotation matrix ${ }^{1 \rightarrow 2} \boldsymbol{M}$ is the matrix of rotation around the axis $\vec{z}$ of angle $-\kappa$ :

$$
\begin{aligned}
& 1 \rightarrow 2 \boldsymbol{M}={ }^{1 \rightarrow 2}\left[\begin{array}{ccc}
\cos (\kappa) & -\sin (\kappa) & 0 \\
\sin (\kappa) & \cos (\kappa) & 0 \\
0 & 0 & 1
\end{array}\right] \quad:=\boldsymbol{M} \\
& { }^{1 \rightarrow 2} \boldsymbol{t}=\quad{ }^{1 \rightarrow 2}\left[\begin{array}{lll}
t_{x} & t_{y} & t_{z}
\end{array}\right] \quad:=\boldsymbol{t}
\end{aligned}
$$

In the following relation, $S(t)$ is the skew-symmetric matrix depending on a 3 -vector $t$ inducing the cross-product.

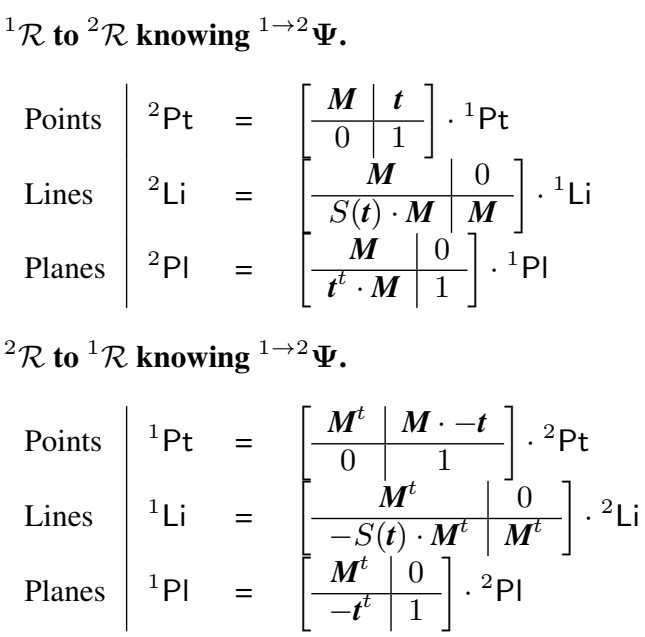

The constraints are defined as follows:

$$
\begin{array}{l|l||l}
\text { Points } & { }^{g} \mathrm{Pt}_{1}^{i}-{ }^{g} \mathrm{Pt}_{2}^{i}=0 & 3 \text { equations } \\
\text { Lines } & { }^{g} \mathrm{Li}_{1}^{i}-{ }^{g} \mathrm{Li}_{2}^{i}=0 \\
\text { Planes } & { }^{g} \mathrm{n}_{1}^{i}={ }^{g} \mathrm{n}_{2}^{i} \text { and }{ }^{g} \mathbf{d}_{1}^{i}={ }^{g} \mathbf{d}_{2}^{i} & 2+1 \text { equations } \\
2+1 \text { equations }
\end{array}
$$

The current definition of the constraints is over parametrized for lines and planes and then brings dependencies between relations. The main issue of these dependencies concerns the estimation of the a posteriori covariance matrix.

\subsection{Design of the system}

Designing the system for bundle adjustment consists in building the Jacobian matrix (matrix of partial derivatives of constraints with respect to unknowns). Using 4 parameters instead of 6 allows us to get a lighter analytical expression of the transformations, inverse transformations and derivatives into Maxima (Shelter, 1998).

The idea is, for pairs of entities, to transform the coordinates of the entities from the station-based frame to a common (or "global") frame and to use one of the two stations as reference to adjust the pose parameters of the second station.

We solve the system using least squares method, defined by the following relation:

$$
\delta \Psi=\left(A^{t} P A\right)^{-1}\left(A^{t} P e\right)
$$

where $\delta \Psi$ is the residual vector, $A$ the Jacobian matrix, $P$ a weight matrix and $e$ the enclosure vector. We add corrections to unknowns and iterate until convergence.

A weight matrix is used, but to properly handle the noise for points, lines and planes, we suggest in subsection 4.1 the use of a common noise factor to define weights.

\section{STUDIES AND ANALYSIS}

In this section, we assess the primitive-based registration approach defined previously. After the description of the simulations, we study two commonly existing configurations into industrial installations: a corridor and a large hall. We compare the possible combinations of objects at several levels of noise. To focus our tests on the performance of the approach, we present here the registration of a simulated noisy data set on a reference data set (pairwise case). All the tests have been implemented using R, (Team, 2011).

\subsection{Simulation of the noise}

The initial data consist in point clouds measured from several stations. 3D primitives are reconstructed and finally entities (points, lines and planes) are extracted. Then, the resulting error on the entities is function of: (a) the scanning error (hardware specifications, scene configuration and scene materials) and (b) the reconstruction error (number of points per reconstructed primitive, size of the visible part of the primitive from the station and performance of the algorithm of reconstruction). The expression of the noise on the entities must make sense with these two consecutive errors. Moreover, to properly handle several types of entities and their combinations, we define a common noise factor, $\sigma_{o}$, that reflects an identical level of uncertainty in the final reconstruction of the entities. $\sigma_{o}$ does not include the polar measurement errors but an average cartesian measurement noise of the point cloud.

For the simulation of the data set, we carried out random sampling of the entities parameters following Gaussian distributions around "ground truth" parameters, as illustrated in figure 3.

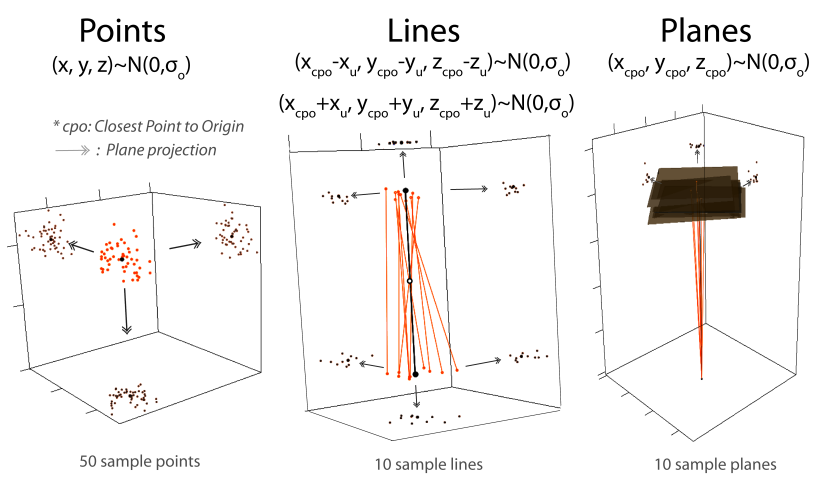

Figure 3: Stochastic models using a common noise factor $\sigma_{o}$. Black: reference entities. Orange: sample interest points and sample entities. Brown: plane projections of sample interest points.

For a point, $\sigma_{o}$ is the standard deviation of a normal distribution around the $(x, y, z)$ coordinates of the reference point. For a line, $\sigma_{o}$ is the standard deviation of a normal distribution around two points of the line: one meter below and one meter above the closest point to origin of the station-based frame. For a plane, $\sigma_{o}$ is the standard deviation of a normal distribution around the $(x, y, z)$ coordinates of the closest point of the plane to the origin of the station-based frame.

3D geometric relations involved in the primitive-based registration are multivariate and analytically complex. Therefore, a complete study of degenerate configurations and numerical instabilities requires strong theoretical improvements. Our tests will not concern an exhaustive analysis, but two configurations commonly encountered in industrial indoor installations. Using approximate 
values of the pose parameters of the station, as introduced in the subsection 3.2, we adjust the pose using primitive-based constraints as presented in the subsection 3.4. We do not study in these examples the influence of wrong matching of $3 \mathrm{D}$ entities.

\subsection{First case: a typical corridor}

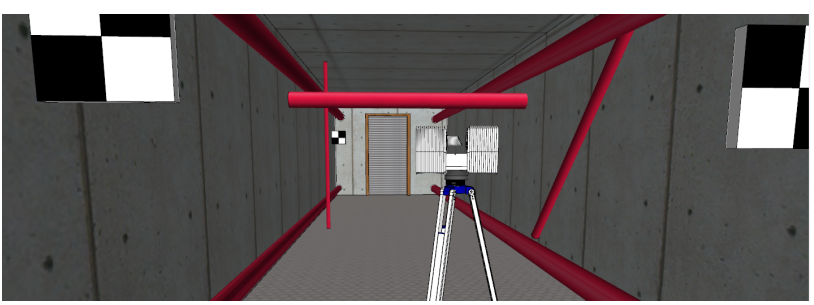

Figure 4: First case: a typical industrial corridor. The scene includes planes, cylinders and targets from which we select 3 points, 7 lines and 5 planes.

The first configuration is a corridor $(8 \mathrm{~m} \times 3 \mathrm{~m} \times 3 \mathrm{~m}$, cf. figure 4$)$, in which we set up 3 points, 7 lines and 4 planes in both the point cloud and the reference data set. These quantities reflect a frequent configuration for corridors. For each possible combination of type of entities and for several level of noise $\sigma_{o}$, we sample 50 scenes around the reference data set.
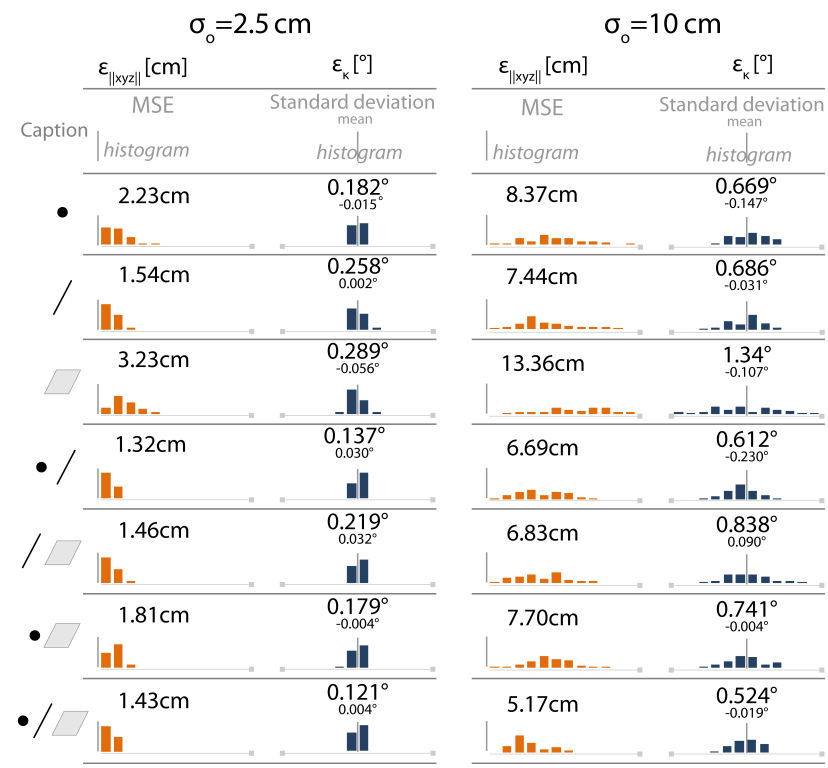

Figure 5: Errors in registration using 3D entities in the case of a corridor. 50 sample tests per case. Orange: error in position. Blue: error in orientation.

For the position, we compute the distance from registered station to reference station for the 50 sample scenes. In figure 5, we display the mean square error and the histogram of position errors for each combination and noise level. Since the orientation is 1dimensional, we compute the error in $\kappa$. The standard deviation, the mean and the histogram of errors in orientation are displayed in figure 5.

We carried out the experiment for 8 values of $\sigma_{o}$ from $0 \mathrm{~cm}$ to $20 \mathrm{~cm}$. For both position and orientation, the pose errors as functions of $\sigma_{o}$ are linear, as expected by the adjustment theory. Consequently, for clarity, we only display two noise levels: $\sigma_{o}=2.5$ $\mathrm{cm}$ and $\sigma_{o}=10 \mathrm{~cm}$. Most of the TLS data acquired for industrial reconstruction purposes correspond to a noise level factor $\sigma_{o}$ between $0.1 \mathrm{~cm}$ and $5 \mathrm{~cm}$.

\subsection{Second case: a large hall}

The second test case is a large hall $(16 \mathrm{~m} \times 16 \mathrm{~m} \times 5 \mathrm{~m})$ in which we set up 2 points, 11 lines and only one plane, the ground. The noise is simulated as previously described. For several levels of noise, we sample 50 scenes and we use all the features available for the registration. The errors of position and orientation as function of $\sigma_{o}$ are also linear, as presented in figure 6 .

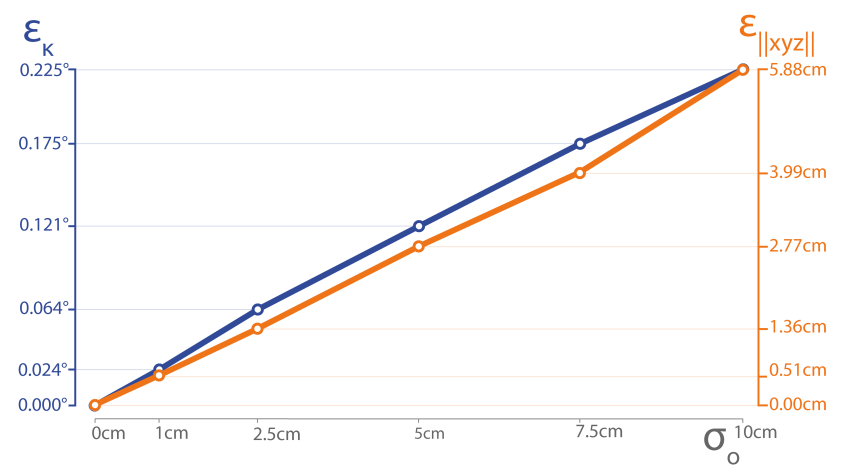

Figure 6: Errors in registration using 3D entities in the case of a hall. 50 sample tests per case. Orange: error in position. Blue: error in orientation.

\subsection{Discussion}

First, the geometric model using constraints on 3D entities presents great results for two commonly seen configurations in industrial indoors. Our simulations highlight interesting properties of the approach: (a) no combination of 3D entities presented clearly larger errors; (b) the largest the number of used 3D entities is, the best are the results as expected; (c) the statistic properties of the errors did not reflect any degenerate configuration in the two presented tests.

However, degenerate configuration may be encountered, such as the trivial case of parallel lines. To avoid numerical instabilities (large enclosure vector and corrections), we recommend to use approximations of the pose parameters using geolocation system. The use of approximations also helps the convergence of the iterations by ensuring the validity of the linearisation in the Jacobian matrix. The use of many 3D entities with diverse directions and positions is the best way to avoid degenerate configurations. The noise of the $3 \mathrm{D}$ entities directly influences the quality of the estimation of the pose parameters.

The quality and the statistical behaviour of the results encourage us to develop the approach. However, since two over an infinity of configurations have been studied, we cannot generalize our results. A first development is the integration of many stations in a block estimation. Traverses, as pairwise series, can be easily handled using our present approach. A network configuration would yet require few adaptations in our method, especially in the consideration of several levels of uncertainty.

The full functionality of our method also requires tools for validating hypothesis on the inputs, such as the exactness of pairing. 


\subsection{Toward a new approach for georeferencing indoor com- plex scenes using external information}

Currently, the method uses as inputs some paired objects and initial approximations of the pose parameters of the stations. As mentioned in subsection 2.3, more information is available and may help estimating the pose parameters. Parts of this information are always available: uncertainty on the approximations of pose parameters, uncertainty on the reconstructed $3 \mathrm{D}$ primitives and uncertainty on the bi-axial compensator. Some other information will be sometimes, not always, available: images and 3D data (maps, previous point cloud or model).

The least squares method outputs adjusted values and cofactors. To help the surveyor making a decision during the acquisition stage, we could imagine other outputs such as probability maps of the solutions.

Current inputs and desired outputs lead us to study the probabilistic approaches to solve the problem of matching and registration, such as bayesian solver for SLAM used in (Montemerlo et al., 2002). The relevance and the use of these probabilistic methods are part of the future work of the authors.

\section{CONCLUSIONS AND FURTHER WORK}

In this article, we have presented a primitive-based registration algorithm using points, lines and planes. These 3D entities are extracted from reconstructed 3D primitives such as sphere, cylinders, planes or targets. The simulations confirmed the validity of the approach for two typical industrial indoor scenes: a corridor and a large hall. This validation is based on the quality of the outputs (accuracy and precision) and on the flexibility of the method (combination of 3D entities). These tests on pairwise cases were necessary to study the possibilities of the approach which can then be extended to more stations using bundle adjustment.

However, the solver does not currently handle external information. Several types of information, such as geolocation systems or a priori CAD models, should be used to guide the estimation of the pose parameters. The relevancy of a probabilistic approach to solve the problem has been discussed.

The authors future work will concern the following points: (a) the integration of multi-sources information; (b) a research of efficient mathematical tools, including probabilistic methods, and (c) the integration of the primitive based registration in the process of TLS data acquisition.

\section{REFERENCES}

Balzani, M., Pellegrinelli, A., Perfetti, N. and Ucceli, F., 2001. A Terrestrial 3D Laser Scanner-Accuracy Tests. In: 18th International Symposium CIPA, Postdam, pp. 445-453.

Barnea, S. and Filin, S., 2008. Keypoint based autonomous registration of terrestrial laser point-clouds. ISPRS Journal of Photogrammetry and Remote Sensing 63(1), pp. 19-35.

Besl, P. J. and Mckay, N. D., 1992. A method for registration of 3-D shapes. IEEE Trans. Pattern Anal. Mach. Intell 14, pp. 239256.

Förstner, W. and Wrobel, B., 2004. Chapter 2 : Mathematical concepts in photogrammetry in: Manual of Photogrammetry, Fifth Edition , pp. 35-53 and 111-165. ASPRS - MC-GLONE, J.-C. edn.
Forsyth, D. A. and Ponce, J., 2002. Computer Vision: A Modern Approach. Us ed edn, Prentice Hall.

Hartley, R. I. and Zisserman, A., 2000. Multiple View Geometry in Computer Vision. Cambridge University Press, ISBN: 0521623049 .

Jaw, J. and Chuang, T., 2008a. Feature-Based Registration Of Terrestrial Lidar Point Clouds. ISPRS Commission III, WG III/3 XXXVII(3b), pp. 303-308.

Jaw, J. and Chuang, T., 2008b. Registration of Ground-Based Lidar Point Clouds By Means Of 3d Line Features. Journal of the Chinese Institute of Engineers 31(6), pp. 1031-1045.

Lichti, D. and Gordon, S., 2004. Error Propagation in Directly Georeferenced Terrestrial Laser Scanner Point Clouds for Cultural Heritage Recording. In: FIG Working Week, WSA2 Modelling and Visualization, Citeseer, Athens, p. 16.

Montemerlo, M., Thrun, S., Koller, D. and Wegbreit, B., 2002. Fastslam: a factored solution to the simultaneous localization and mapping problem. In: Eighteenth national conference on Artificial intelligence, American Association for Artificial Intelligence, pp. 593-598.

Nüchter, A., Elseberg, J., Schneider, P. and Paulus, D., 2010. Study of parameterizations for the rigid body transformations of the scan registration problem. Computer Vision and Image Understanding 114(8), pp. 963-980.

Rabbani, T. and van den Heuvel, F., 2005. Automatic point cloud registration using constrained search for corresponding objects. In: H. Kahmen and A. Gruen (eds), Proceedings of 7th Conference on Optical, Vol. 1, Vienna, Austria, pp. 3-5.

Rabbani, T., Dijkman, S. T., Van Den Heuvel, F. and Vosselman, G., 2007. An integrated approach for modelling and global registration of point clouds. ISPRS Journal of Photogrammetry and Remote Sensing 61(6), pp. 355-370.

Reshetyuk, Y., 2009. Self-calibration and direct georeferencing in terrestrial laser scanning. PhD thesis, Royal Institute of Technology, Stockholm, Sweden.

Scaioni, M., 2005. Direct georeferencing of TLS in surveying of complex sites. In: Proceedings of the ISPRS Working Group V/4 Workshop 3D-ARCH "Virtual Reconstruction and Visualization of Complex Architectures", Mestre-Venice, Italy.

Schenk, T., 2004. From point-based to feature-based aerial triangulation, 1. Introduction . ISPRS Journal of Photogrammetry and Remote Sensing 58(5-6), pp. 315-329.

Schnabel, R., Wahl, R. and Klein, R., 2007. Efficient RANSAC for Point-Cloud Shape Detection. Computer Graphics Forum 26(2), pp. 214-226.

Shelter, W., 1998. Maxima, a computer algebra system, version 5.18.1 (2009). http://maxima. sourceforge. net/.

Team, R. D. C., 2011. R: A Language and Environment for Statistical Computing. R Foundation for Statistical Computing, Vienna, Austria. ISBN 3-900051-07-0. http: //www.R-project. org.

Thapa, A., 2009. Semantic Feature Based Registration of Laser Point Clouds. Master thesis, International Institute For Geo-Information Science And Earth Observation Enschede, The Netherlands. 